\title{
Utility of FOS as diagnostic marker for osteoid osteoma and osteoblastoma
}

\author{
Suk Wai Lam ${ }^{1} \cdot$ Arjen H. G. Cleven ${ }^{1} \cdot$ Herman M. Kroon ${ }^{2} \cdot$ Inge H. Briaire-de Bruijn ${ }^{1} \cdot$ Karoly Szuhai $^{3}$. \\ Judith V. M. G. Bovée ${ }^{1}$ (D)
}

Received: 1 August 2019 / Revised: 24 September 2019 / Accepted: 30 September 2019 / Published online: 25 November 2019

(C) The Author(s) 2019

\begin{abstract}
Osteoid osteoma and osteoblastoma are bone-forming tumors shown to harbor $F O S(87 \%)$ and $F O S B(3 \%)$ rearrangements. The aim was to evaluate the immunohistochemical expression of FOS and FOSB in these tumors in comparison to other bone tumors, to evaluate the influence of decalcification, and to correlate immunohistochemical findings with the underlying genetic alteration using fluorescence in situ hybridization (FISH). Immunohistochemistry using whole sections was performed on osteoid osteoma $(n=23)$, osteoblastoma $(n=22)$, osteoblastoma-like osteosarcoma $(n=3)$, reactive $(n=3)$, and proliferative $(n=11)$ bone lesions. Immunoreactivity in giant cell tumor of bone $(n=74)$, aneurysmal bone cyst $(n=6)$, chondromyxoid fibroma $(n=20)$, osteosarcoma $(n=85)$, chondroblastoma $(n=17)$, and clear cell chondrosarcoma $(n=20)$ was assessed using tissue micro arrays. Strong nuclear expression of FOS in $>50 \%$ of the tumor cells was observed in all osteoid osteomas (22/22), in 57\% of osteoblastomas $(12 / 21)$ and in 3/197 control cases. FOS immunoreactivity disappeared after $>3$ days decalcification. FOS rearrangements were present in $94 \%$ of osteoid osteomas and osteoblastomas, with a concordance of $86 \%$ between FISH and immunohistochemistry. Two osteoblastomas (5\%) were positive for FOSB, as opposed to 8/177 control cases. Additional FISH revealed no FOSB rearrangements in these cases. To conclude, in short decalcified biopsies, FOS immunohistochemistry can be used to diagnose osteoid osteoma and osteoblastoma, as overexpression is seen in the majority, being rare in their mimics. FOS immunohistochemistry should not be used after long decalcification. Moreover, low level of focal expression found in other lesions and tissues might cause diagnostic problems, in which case FISH could be employed.
\end{abstract}

Keywords Osteoid osteoma · Osteoblastoma $\cdot$ Immunohistochemistry $\cdot$ Fluorescence in situ hybridization $\cdot$ FOS $\cdot$ Bone tumors

\section{Introduction}

Osteoid osteoma and osteoblastoma are common boneforming tumors and typically present during the second

Electronic supplementary material The online version of this article (https://doi.org/10.1007/s00428-019-02684-9) contains supplementary material, which is available to authorized users.

Judith V. M. G. Bovée

j.v.m.g.bovee@lumc.nl

1 Department of Pathology, Leiden University Medical Center, Albinusdreef 2, 2333, ZA Leiden, The Netherlands

2 Department of Radiology, Leiden University Medical Center, Albinusdreef 2, 2333, ZA Leiden, The Netherlands

3 Department of Cell and Chemical Biology, Leiden University Medical Center, Einthovenweg 20, 2333, ZC

Leiden, The Netherlands decade of life. They have no malignant potential, but osteoblastoma can behave locally aggressive $[1,2]$. Both lesions are more or less histologically indistinguishable, and distinction is predominantly based on size (diameter below or above $2 \mathrm{~cm}$, respectively) [3]. In addition, osteoid osteomas are usually located in the long bones and present with nocturnal pain relieved by nonsteroidal anti-inflammatory drugs (NSAIDs), while osteoblastomas have a preference for the posterior column of the spine. The most essential feature in osteoid osteoma is the radiographic presence of a central lucent area (nidus), which is surrounded by dense sclerotic bone tissue. In the nidus, regular trabeculae of woven bone are present. These trabeculae are lined by active osteoblasts with vascularized stroma in between. In osteoblastoma, the distribution of woven bone can be slightly less organized, as compared to the nidus of an osteoid osteoma. In the past years, deep sequencing has rapidly advanced the field, as it has provided increased knowledge on the molecular background of 
bone tumors. Based on these findings, molecular testing as well as specific immunohistochemistry has found its way in routine bone tumor diagnostics that historically heavily relied on morphology and has improved diagnostic accuracy [4, 5]. Recently, recurrent translocations in FOS (87\%) and FOSB (3\%) were found in osteoblastoma and osteoid osteoma [6]. Both FOS and FOSB are part of the FOS family of transcription factors and were shown to play a role in diverse biological processes, including osteoblast differentiation and proliferation [7]. Also, similar rearrangements are found in vascular tumors [8-11]. The aim of this study was to evaluate whether the recently found FOS and FOSB rearrangements can be used as an auxiliary diagnostic tool in routine bone tumor diagnosis. We compared immunohistochemistry of FOS and FOSB between osteoid osteoma and osteoblastoma and other lesions with bone deposition. We evaluated the influence of decalcification and, in addition, correlated the immunohistochemical findings to the underlying genetic alteration using interphase fluorescence in situ hybridization (FISH).

\section{Materials and methods}

\section{Sample collection}

Whole sections were cut from osteoid osteoma $(n=23)$ and osteoblastoma $(n=22)$. All cases were retrieved from the Department of Pathology at Leiden University Medical Center, with the exception of one osteoblastoma. Since samples were collected from routinely processed diagnostic cases, fixation and decalcification time varied. For all internal cases, samples were decalcified in formic acid for a short period of $4 \mathrm{~h}$ or longer (range: $2-15$ days) until ready for cutting. For the external cases, exact decalcification time and procedure were unknown. The majority (21/23) of osteoid osteoma samples were decalcified shortly, while for osteoblastoma samples, the decalcification time was more variable.

For comparison we included whole sections of proliferative bone lesions (subungual exostosis $(n=3)$, bizarre parosteal osteochondromatous proliferation (BPOP) $(n=5)$, and myositis ossificans $(n=3)$ ), samples with reactive callus formation $(n=3)$, and osteoblastoma-like osteosarcoma $(n=3)$. Furthermore, sections of previously constructed tissue micro arrays (TMAs) of giant cell tumor of bone $(n=74)$ [12], aneurysmal bone cyst $(n=6)$ [12], chondromyxoid fibroma $(n=13)$ [12], osteosarcoma $(n=76)[12,13]$, chondroblastoma $(n=11)$ [12], and clear cell chondrosarcoma $(n=13)$ were evaluated [14]. In addition, TMAs of chondroblastoma $(n=6)$ and chondromyxoid fibroma $(n=7)$ and osteosarcoma $(n=8)$ were constructed as described previously [12]. For each sample, three $1.5 \mathrm{~mm}$ cores were present on the TMA for representativity. For the osteosarcoma TMA, samples from a previously published cohort were used [15]. For osteosarcoma, both biopsies ( $n=13)$ as well as resection specimens were used. As far as could be retrieved, among the osteosarcoma cases, there were samples that were not $(n=13)$ or short $(<3$ days, $n=19)$ decalcified.

\section{Decalcification}

A decalcification series of placental tissue was taken along with different decalcification periods, ranging from $4 \mathrm{~h}$ to 14 days, using a similar protocol as for internal diagnostic samples. Samples were decalcified using $98-100 \%$ formic acid (Merck, Kenilworth, New Jersey, USA), diluted 1:5 in demi water with $2.6 \%$ sodium formate.

\section{Immunohistochemistry}

Immunohistochemistry was performed as described previously with minor adjustments [12]. In brief, microwave antigen retrieval was performed in Tris-EDTA ( $\mathrm{pH}$ 9.0). A rabbit polyclonal antibody was used against the N-terminal region of FOS (clone, F7799, Sigma, St. Louis, Missouri, USA) and a rabbit monoclonal antibody for FOSB (clone, 5G4, Cell Signaling Technology, Danvers, Massachusetts, USA). Sections for FOS staining were pre-incubated with PBS/1\% BSA $/ 5 \%$ nonfat dry milk for $30 \mathrm{~min}$ at room temperature. Primary antibody was diluted in PBS/1\% BSA at 1:200,000 for FOS and at 1:30,000 for FOSB, after which slides were incubated overnight at $4{ }^{\circ} \mathrm{C}$. Placenta served as a positive control.

\section{Evaluation of staining}

Slides were scored by two observers independently (SWL and JVMGB). Immunoreactivity was scored according to the intensity of the staining ( $1=$ weak, $2=$ moderate, or $3=$ strong $)$ and the percentage of tumor cells (i.e., the plump osteoblastic cells lining the osteoid or bone) with nuclear staining $(1=1-$ $25 \%, 2=26-50 \%, 3=51-75 \%$, and $4=76-100 \%$ ) [16]. Positivity was defined as strong nuclear staining in more than $50 \%$ of the tumor cells. For the tumors on the TMAs, an average score of 3 cores was used for analysis.

\section{Interphase fluorescence in situ hybridization}

For in situ hybridization, BAC probes were used proximal and distal to $F O S$ and $F O S B$, as described previously $[8,17]$. In first instance, a two-color FISH using FOS break-apart and $F O S B$ break-apart was performed on FFPE sections. In case of no signal due to long decalcification, frozen sections were used as an alternative whenever possible. FISH on FFPE slides and on frozen material was performed as described previously $[8,17,18]$. FISH for FOS was performed for all osteoid osteomas and osteoblastomas. In case no FOS 
rearrangement was present, FISH for FOSB followed. Also, for the immunohistochemically positive control cases, additional FISH was performed. Slides were scored by two observers independently (SWL, KS).

\section{Results}

\section{Clinicopathological features of osteoid osteoma and osteoblastoma}

Osteoid osteoma and osteoblastoma cases were diagnosed at our institution in a multidisciplinary setting. The average age for osteoid osteoma patients was 22 years (range, 8 to 69 years) and 23 years for osteoblastoma patients (range, 4 to 50 years). In both groups, males were overrepresented (male to female ratio of 3.6:1 and 3.8:1, respectively). For osteoid osteomas, long bones of the lower extremity were affected most (femur, $n=9$; tibia, $n=3$ ), while seven osteoblastomas $(30 \%)$ were located in the vertebral column.

All osteoid osteoma specimens showed classic morphology and were composed of trabeculae of woven bone. Surrounding osteoblasts were small, with monomorphic oval nuclei and a moderate amount of cytoplasm. Occasionally, few osteoblasts with more abundant eosinophilic cytoplasm were dispersed throughout the specimen. Osteoclast-like giant cells were present in all cases (Fig. 1). The morphology of osteoblastoma cases was more variable. Three cases showed a clear epithelioid morphology, defined as the presence of numerous large osteoblasts with abundant eosinophilic cytoplasm, in the majority of the specimen. Nuclei were enlarged and were more irregular and hyperchromatic (Fig. 2). Osteoblastoma was distinguished from osteoblastoma-like osteosarcoma, as the latter demonstrated malignant radiological features and presence of infiltrative growth (Fig. 2).

\section{Immunohistochemistry of FOS and FOSB}

Strong and diffuse nuclear staining for FOS was observed in all osteoid osteomas (22/22), in $57 \%$ of the osteoblastomas (12/21) (Fig. 1), and in one case with reactive callus formation (1/3). All three osteoblastoma-like osteosarcomas were negative (Fig. 2). Moderate staining in $>50 \%$ of the tumor cells was seen in additional 3 osteoblastomas, 1 (out of 54) conventional osteosarcoma, and 1 (out of 6) aneurysmal bone cyst (Supplementary Table 1). Moreover, $45 \%$ of the proliferative bone lesions ( $3 / 5$ BPOP, $1 / 3$ subungual exostosis, and $1 / 3$ myositis ossificans) showed moderate staining in $>50 \%$ of the osteoblast-like cells (Fig. 3). Evaluation of 180 samples on TMAs revealed two positive osteosarcomas, which had an osteoblastic and sclerosing morphology (Fig. 4). All other tumors did not show strong and diffuse staining (Table 1). Thus, in total only 3 out of 197 of other bone-forming tumors were positive for FOS immunohistochemistry. The surrounding normal tissues also showed variable moderate to strong nuclear staining, such as endothelial cells and pericytes of mainly larger vessels, striated muscle, chondrocytes, and the epidermis (Supplementary Fig. 1). Osteoclasts were completely negative or showed membranous or cytoplasmic staining.

Two of 21 osteoblastomas were FOSB positive (in addition to FOS), while all 22 osteoid osteomas, all 3 osteoblastomalike osteosarcomas, and both cases with reactive callus formation were negative. Five proliferative bone lesions (4/5 BPOP and $1 / 3$ myositis ossificans) showed strong and diffuse nuclear staining. Three out of the 164 TMA samples were positive (osteosarcoma $(n=1)$ and giant cell tumors of bone $(n=2)$ ), while the remaining samples were negative (Table 1). Taken together, $8 / 177$ of other bone-forming tumors were FOSB positive.

\section{Effect of decalcification on FOS and FOSB immunoreactivity}

Nuclear staining of decidual cells in placental tissue was moderate to strong in non-decalcified placenta, in short decalcified placenta, and in decalcified placenta up to 3 days. After 3 days, nuclear staining diminished for FOS, turning almost negative after 14 days (Supplementary Fig. 2). For FOSB immunohistochemistry, this phenomenon was not observed as after 14 days of decalcification strong nuclear reactivity was retained (Supplementary Fig. 3).

\section{Correlation of immunohistochemistry with FISH}

Interphase FISH for FOS was performed for all osteoid osteomas and osteoblastomas, which was successful in 31/43 cases (27 paraffin, 4 frozen sections). FOS rearrangements were present in $94 \%(n=29)$ of cases, of which $86 \%(n=25)$ correlated to strong overexpression of FOS at immunohistochemistry (Table 2). Four cases were translocation positive, while immunohistochemistry was scored negative; they displayed moderate staining in a variable percentage of cells, which was below the cutoff we used. All four cases were osteoblastomas, of which decalcification time could not be tracked down or varied between $4 \mathrm{~h}$ and 2 days.

Two cases lacked FOS rearrangement by FISH, while one of these was immunohistochemically positive (Table 2). To rule out cross-reactivity of the FOS antibody, additional FISH for FOSB rearrangement was performed, which was negative. After review of H\&E slides, clinical records, and radiology, the diagnosis of osteoblastoma remained unchanged (Supplementary Figs. 4 and 5). FOS rearrangements were absent in other bone-forming lesions with strong expression of FOS and for which we were able to perform FISH (osteosarcoma $(n=1)$ and reactive bone with callus formation 

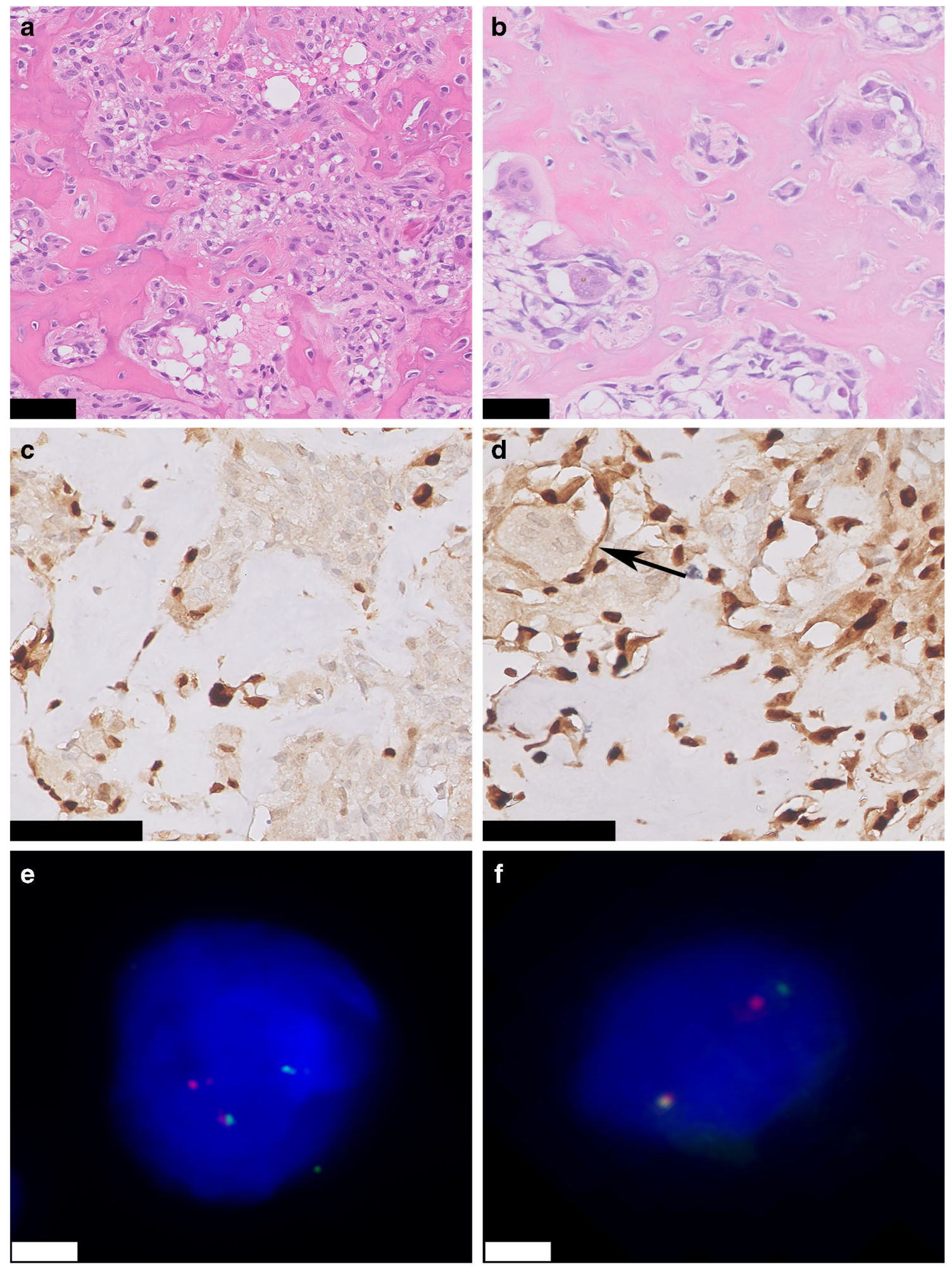

Fig. 1 Osteoid osteoma and osteoblastoma. Hematoxylin \& Eosin (H\&E) staining of the nidus of osteoid osteoma a and osteoblastoma $\mathbf{b}$. Regular deposited trabeculae of woven bone are surrounded by active osteoblasts. Both osteoid osteoma and osteoblastoma show strong and diffuse nuclear expression of FOS. Osteoclast-like giant cells are negative (arrow) $\mathbf{c}$ and

d. Fluorescence in situ hybridization (FISH) using split-apart probes for FOS shows a segregated red and green signal in both osteoid osteoma and osteoblastoma, indicating a FOS rearrangement e and f. Scale bar, $50 \mu \mathrm{m}$ a-d. Scale bar, $5 \mu \mathrm{m} \mathbf{e}$ and $\mathbf{f}$ 

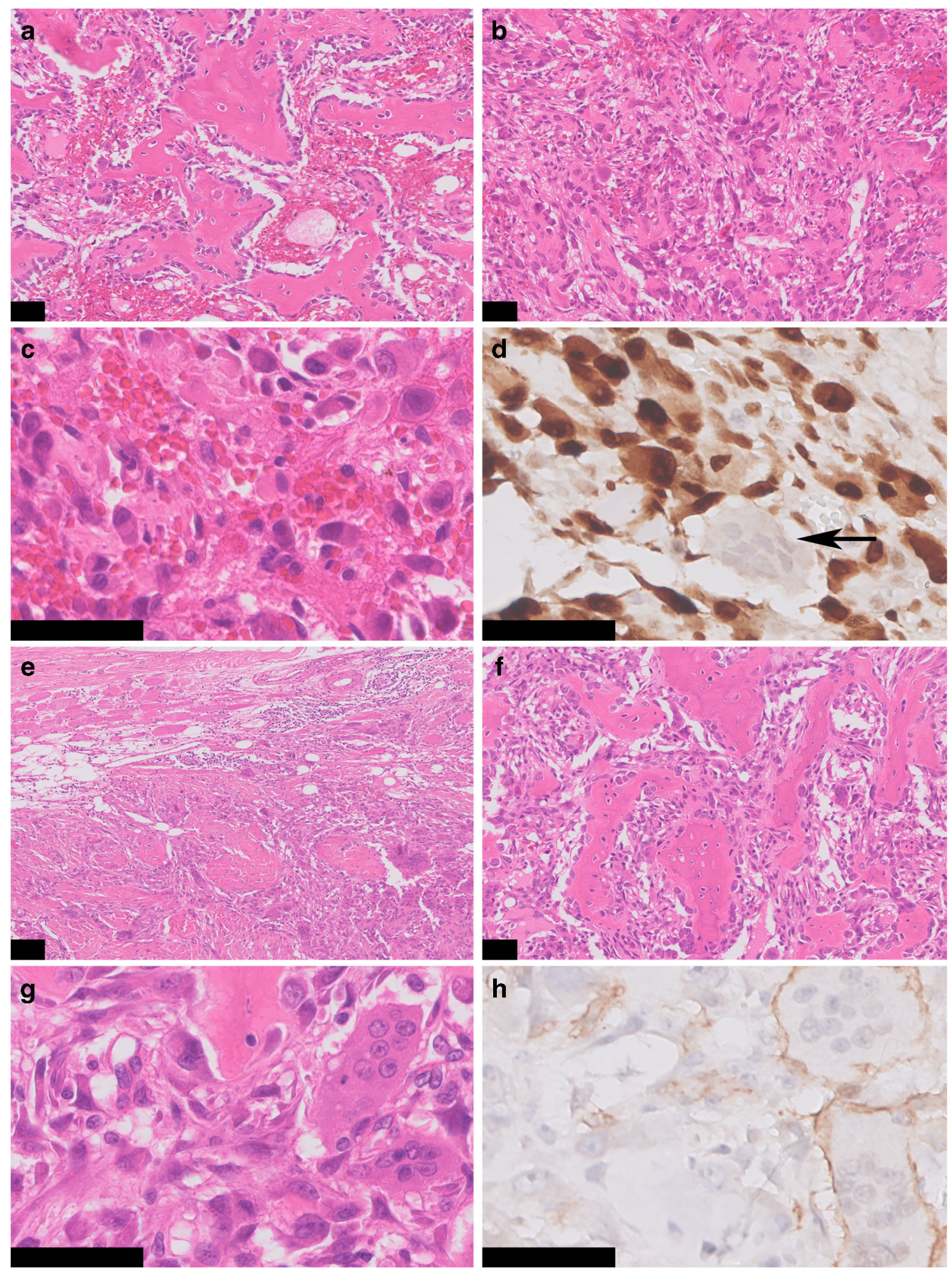

Fig. 2 Epithelioid osteoblastoma and osteoblastoma-like osteosarcoma. $H \& E$ staining of an epithelioid osteoblastoma shows maturation with presence of trabeculae of woven bone, while the central area shows less osteoid deposition a and $\mathbf{b}$. Numerous large, plump osteoblasts with abundant eosinophilic cytoplasm are scattered throughout the specimen. Atypia can be frequently encountered, with osteoblasts harboring hyperchromatic and irregular enlarged nuclei, which may resemble osteosarcoma c. FOS immunohistochemistry showing diffuse and

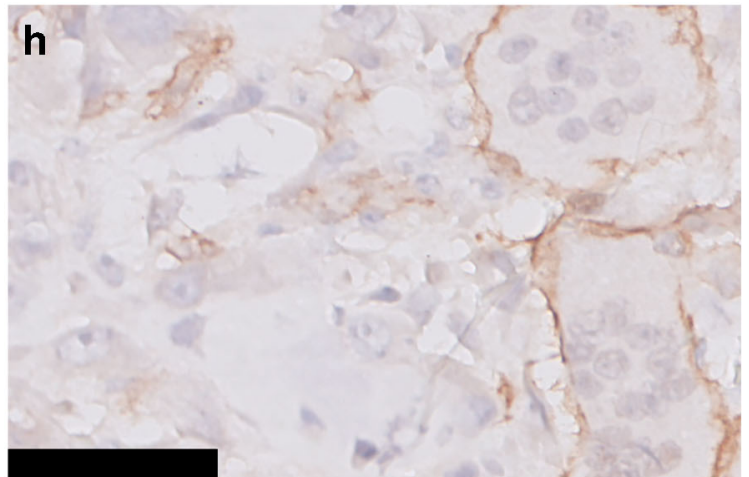

strong nuclear staining in all osteoblasts. Osteoclasts-like giant cells are negative (arrow) d. Osteoblastoma-like osteosarcoma with extensive soft tissue involvement (H\&E) e. Tumor cells show an epithelioid aspect with enlarged nuclei with a prominent nucleolus. Note the trabeculae of neoplastic woven bone, mimicking osteoblastoma $\mathbf{f}$ and $\mathbf{g}$. FOS immunohistochemistry is negative $\mathbf{h}$. Scale bar, $100 \mu \mathrm{m}$ a and e. Scale bar, $50 \mu \mathrm{m} \mathbf{b}-\mathbf{d}$ and $\mathbf{f}-\mathbf{h}$ 

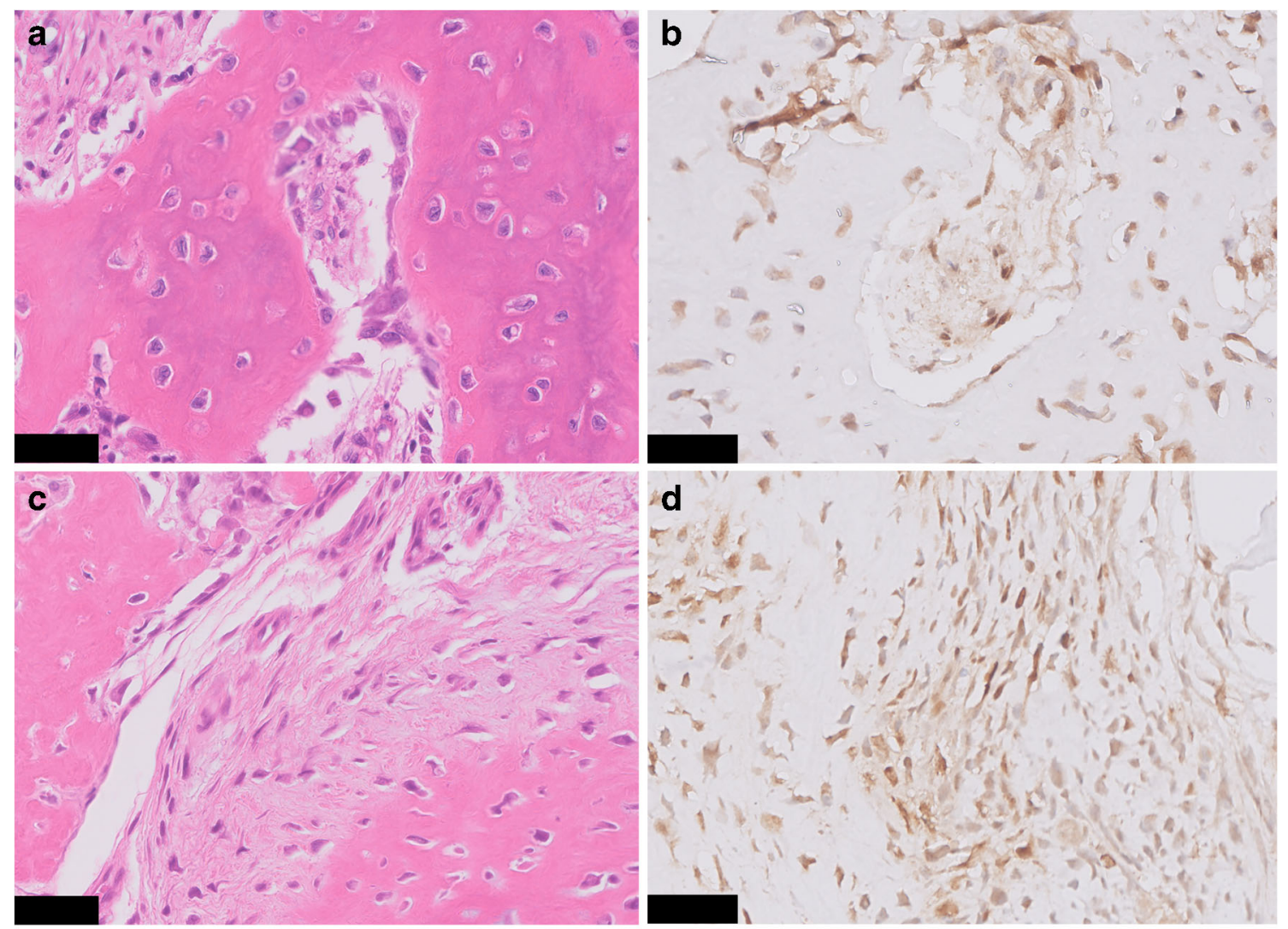

d
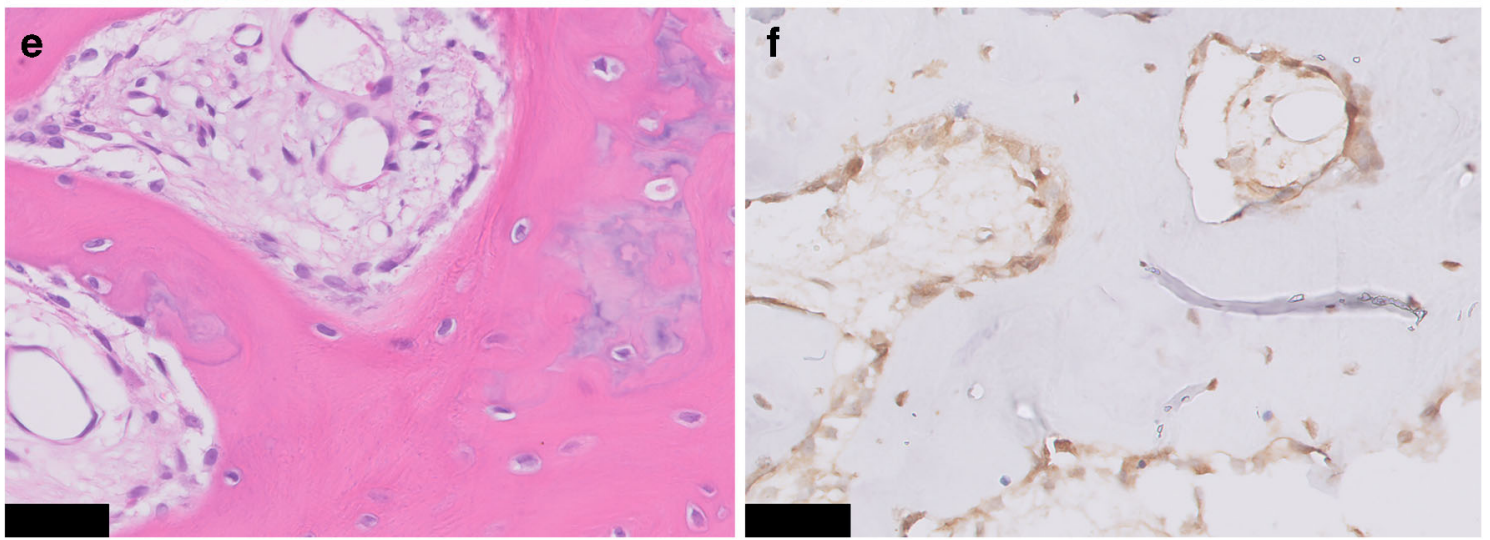

Fig. 3 FOS immunohistochemistry in proliferative bone lesions. Myositis ossificans, with peripheral zone showing ill-defined trabeculae of woven bone, rimmed with osteoblasts (H\&E) a. Immunohistochemistry of FOS showing moderate nuclear staining of osteoblasts b. Bizarre parosteal osteochondromatous proliferation with a disorganized mix of woven bone

and spindle cells (H\&E) c, where additional FOS immunohistochemistry shows moderate staining in both components $\mathbf{d}$. Central area with trabecular bone in subungual exostosis $(\mathrm{H} \& \mathrm{E}) \mathbf{e}$, showing moderate expression of FOS in osteoblasts f. Scale bar, $50 \mu \mathrm{m}(\mathbf{a}-\mathbf{f})$

$(n=1))$. Of note, the osteosarcoma sample showed multiple copies of the FOS-locus (Fig. 4).

In addition, FISH for FOSB was performed in 10 cases with strong immunoreactivity for FOSB. FOSB rearrangements were absent in 5 cases in which FISH was successful (BPOP $(n=1)$, osteosarcoma $(n=1)$, giant cell tumor of bone $(n=2)$, and osteoblastoma $(n=1))$. Of these, the osteoblastoma case already showed a FOS rearrangement. The osteosarcoma case showed, in addition to multiple copies of the FOS-locus, also multiple copies of the FOSB-locus (Fig. 4). Both giant

cell tumors of bone cases harbored H3F3A p.Gly34Trp mutations shown in a previous study [12].

\section{Discussion}

In this study, we evaluated the utility of the use of immunohistochemistry of FOS to diagnose osteoid osteoma and osteoblastoma. So far this has only been tested in small series, with divergent results, as positivity ranged from $0(n=11)$ to 
Fig. 4 High-grade osteoblastic osteosarcoma. H\&E staining shows atypical tumor cells depositing lace-like osteoid (H\&E) (A).

Immunohistochemistry for FOS shows moderate to strong nuclear staining of the tumor cells (B). Additional FISH for $F O S$ and FOSB shows gains of the FOSand $F O S B$-locus, respectively $(\mathbf{C}$ and D). Scale bar, $50 \mu \mathrm{m}$ (A and B). Scale bar, $5 \mu \mathrm{m}$ (C and D)
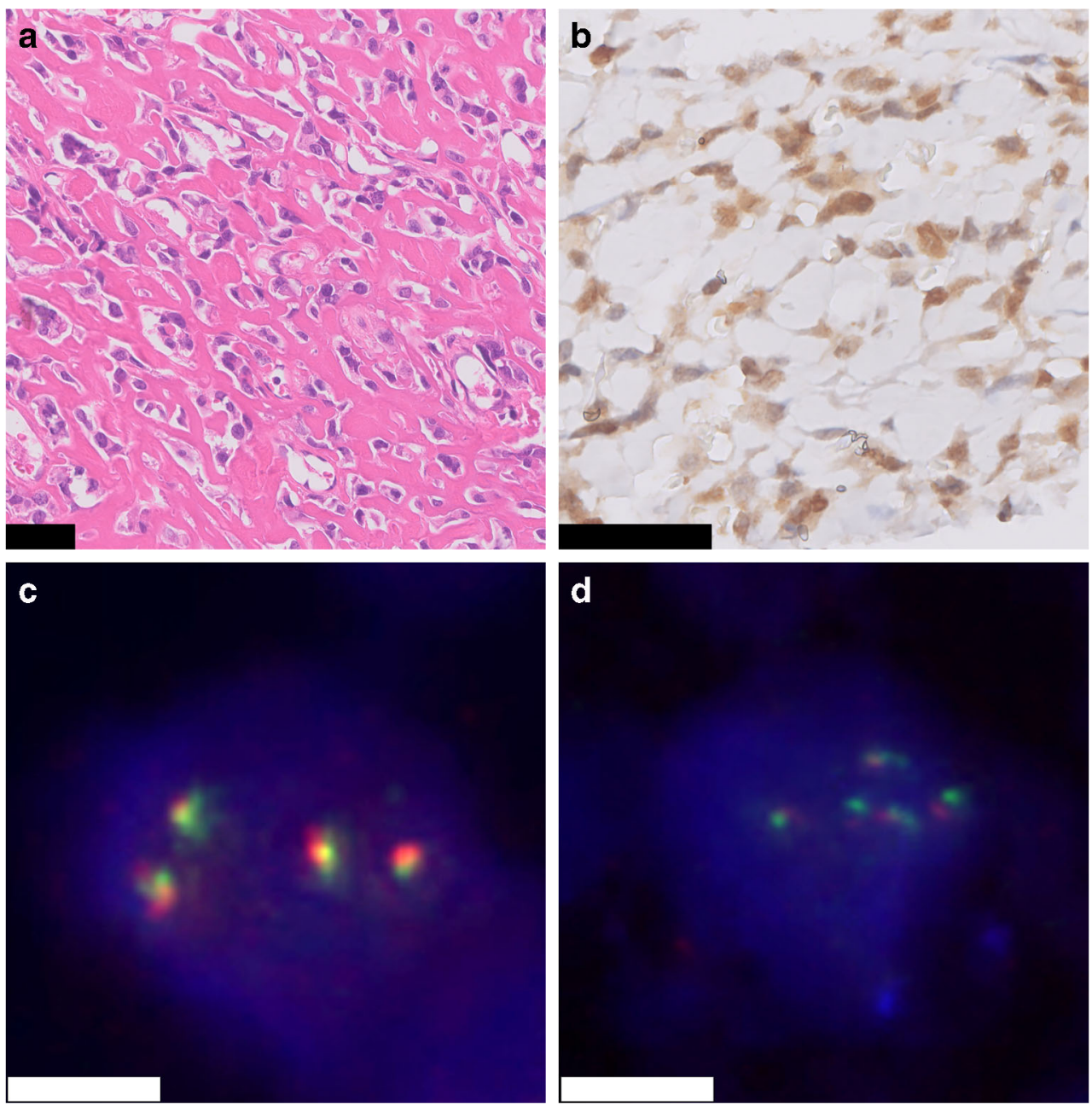

$100 \%(n=3)[6,19]$. Strong overexpression of FOS at immunohistochemistry correlated strongly with the underlying FOS rearrangement. While in a previous study in a minority of cases, FOSB rearrangements were present, instead of FOS rearrangements [6], we did not find any, rendering FOSB immunohistochemistry diagnostically not relevant. Our study indicates that there are two important caveats that pathologists should be aware of when applying immunohistochemistry for FOS to diagnose osteoid osteoma and osteoblastoma.

First, we showed that after $>3$ days of acid-based decalcification, immunoreactivity for FOS disappeared. Though decalcification in EDTA preserves DNA and immunogenicity, acidbased solutions are still commonly used and may affect antigen preservation, leading to loss of sensitivity of immunohistochemistry [20]. In this study, a striking difference between osteoid osteoma and osteoblastoma samples for FOS expression was noticed, as all osteoid osteoma, but only $57 \%$ of osteoblastomas showed positivity. In general, osteoid osteoma samples were all small and were decalcified for a short period of only $4 \mathrm{~h}$ in most cases, as opposed to osteoblastoma samples. The additional decalcified placental series confirmed diminished nuclear staining after a longer period of decalcification for FOS, while FOSB remained intact. Thus, long decalcification times specifically affect FOS immunohistochemistry, and immunohistochemistry should not be used on resection specimens after prolonged acid-based decalcification.

Second, we scored FOS overexpression as strong and diffuse ( $>50 \%$ of tumor cells) nuclear expression that we found in all 22 osteoid osteomas and in 12 of 21 osteoblastomas. As could be expected based on their role in normal osteoblast maturation and differentiation [7], we noticed moderate to strong nuclear positivity for FOS and FOSB in the areas of bone deposition in several reactive and proliferative boneforming lesions. Of the neoplasms, only 1 of 6 aneurysmal bone cysts showed moderate staining in $>50 \%$ of the tumor cells, while this was absent in other tumors. This can be a pitfall when using immunohistochemistry, necessitating confirmation by FISH under these circumstances. Partial weak staining was noticed in the majority of other samples and should be considered as not representative of translocationinduced overexpression. Moreover, consistent with previous findings in which copy number gains were noticed in FOS immunopositive osteosarcoma [6], we also observed FOS positivity in two osteosarcoma samples (osteoblastic and 
Table 1 Summary of immunohistochemistry for FOS and FOSB

\begin{tabular}{lll}
\hline Tumor type & $\begin{array}{l}\text { FOS } \\
\text { positive* }(\%)\end{array}$ & $\begin{array}{l}\text { FOSB } \\
\text { positive* }(\%)\end{array}$ \\
\hline Osteoid osteoma & $22 / 22(100)$ & $0 / 22$ \\
Osteoblastoma & $12 / 21(57)$ & $2 / 21(10)$ \\
Conventional osteosarcoma & $2 / 54(4)$ & $1 / 55(2)$ \\
Giant cell tumor of bone & $0 / 73$ & $2 / 72(3)$ \\
Aneurysmal bone cyst & $0 / 6$ & $0 / 6$ \\
Chondromyxoid fibroma & $0 / 19$ & $0 / 19$ \\
Chondroblastoma & $0 / 14$ & $0 / 15$ \\
Clear cell chondrosarcoma & $0 / 17$ & - \\
Reactive bone with callus formation & $1 / 3(33)$ & $0 / 2$ \\
Proliferative bone lesion: & $0 / 11$ & $5 / 8(63)$ \\
Subungual exostosis & $0 / 3$ & - \\
Bizarre parosteal & $0 / 5$ & $4 / 5(80)$ \\
$\quad$ osteochondromatous proliferation & & $1 / 3(33)$ \\
Myositis ossificans & $0 / 3$ & \\
\hline
\end{tabular}

*defined as strong nuclear expression in $>50 \%$ of tumor cells

sclerosing subtype). In one case, FISH was possible, which showed gains of $F O S$ and $F O S B$, potentially resulting in overexpression at immunohistochemistry.

The FOS transcription factor family includes FOS, FOSB, FOSL1, and FOSL2 and encodes leucine zipper proteins that can dimerize with proteins of the JUN family, thereby forming the transcription factor complex AP-1. This way, the FOS proteins regulate a diverse array of biological processes, including cell proliferation, differentiation, and survival. Functional studies have shown that FOS and FOSB, together with other family members of FOS family, are highly expressed during normal osteoblast maturation [21]. Retroviral FOS oncogene can cause osteosarcoma in mouse model systems, when fused with a highly active promoter and the $\mathrm{v}-$ fos 3 ' untranslated region [22].

Similar rearrangements of $F O S$ and $F O S B$ were previously found in vascular tumors [8-11]. Identical to FOS-rearranged epithelioid hemangioma, the translocations involve various genes or intergenic regions and lead to a premature stop codon, at or early after the break points that always involve exon 4 of FOS $[6,8]$. This causes loss of the C-terminal end of the

Table 2. Correlation between FOS immunohistochemistry and interphase fluorescence in situ hybridization in osteoid osteoma and osteoblastoma

\begin{tabular}{lll}
\hline FOS & Translocation + & Translocation - \\
\hline Immunohistochemistry + & 25 & 1 \\
Immunohistochemistry - & 4 & 1 \\
\hline
\end{tabular}

+ , positive; -, negative protein, rendering the protein resistant to degradation causing high expression in tumor cells [23]. The FOSB fusions described in atypical epithelioid hemangioma and pseudomyogenic hemangioendothelioma occur at the Nterminal part of the protein and are most likely induced by promoter swap events, causing upregulation of FOSB $[10$, $11,24]$.

For bone tumor pathologists, a challenging diagnostic problem is to discriminate epithelioid osteoblastoma from high-grade osteoblastic osteosarcoma. Epithelioid osteoblastomas can be composed of large, plump osteoblasts, surrounded by abundant eosinophilic cytoplasm. Additional degenerative nuclear atypia can be present, accompanied by mitotic figures. Similarly, osteoblastoma-like osteosarcoma can mimic osteoblastoma. Distinction is of crucial importance, as prognosis and treatment differ significantly. While infiltration of host bone and lack of differentiation towards the periphery seem to be the most discriminating features between (epithelioid) osteoblastoma and (osteoblastoma-like) osteosarcoma, this is not often assessable in biopsy and curettage specimens $[25,26]$. Although numbers are small, our present results indicate that immunohistochemistry and/or FISH for FOS can be of help in distinguishing (epithelioid) osteoblastoma from osteosarcoma, especially since there are no specific antibodies or molecular tests for osteosarcoma.

To summarize, FOS immunohistochemistry can be used as an auxiliary tool for osteoid osteoma and osteoblastoma in short decalcified tissue, while FOSB immunohistochemistry is diagnostically not useful. However, FOS immunohistochemistry should not be used after long decalcification, and the low-level focal expression found in other lesions and tissues, especially reactive bone, might be confusing. Under these circumstances, the use of FISH for FOS could be diagnostically useful, for cases where it is difficult to distinguish osteoid osteoma and osteoblastoma from their histologic mimics.

Acknowledgments We thank D.G.P van IJzendoorn for providing $F O S B$ probes for FISH. M.L Kuijjer, Y.T. Sundara, M. Kostine, D. Meijer, B. Liegl-Atzwanger, N. Athanasou, P. Picci, and S. Daugaard are acknowledged for the contribution to previous constructed TMAs.

Author contributions The study was designed, written, and reviewed by S.W. Lam and J.V.M.G. Bovée. All authors contributed to the data collection, data analysis, and interpretation. The manuscript was approved by all authors.

\section{Compliance with ethical standards}

Ethical approval All samples were handled according to the ethical guidelines described in "Code for Proper Secondary Use of Human Tissue in the Netherlands" in a coded (pseudonymized) manner, as approved by the Leiden University Medical Center ethical board (B17.036 and B19.007). 
Open Access This article is distributed under the terms of the Creative Commons Attribution 4.0 International License (http:// creativecommons.org/licenses/by/4.0/), which permits unrestricted use, distribution, and reproduction in any medium, provided you give appropriate credit to the original author(s) and the source, provide a link to the Creative Commons license, and indicate if changes were made.

\section{References}

1. Jaffe HL (1953) Osteoid-osteoma. Proc R Soc Med 46:1007-1012

2. Jaffe HL (1935) "Osteoid-osteoma": a benign osteoblastic tumor composed of osteoid and atypical bone. Arch Surg 31:709-728. https://doi.org/10.1001/archsurg.1935.01180170034003

3. Schajowicz F, Lemos C (1970) Osteoid osteoma and osteoblastoma. Closely related entities of osteoblastic derivation. Acta Orthop Scand 41:272-291

4. Lam SW, van Jzendoorn DGP, Cleton-Jansen AM, Szuhai K, Bovee JVMG (2019) Molecular pathology of bone tumors. J Mol Diagn 21:171-182. https://doi.org/10.1016/j.jmoldx.2018.11.002

5. Baumhoer D, Amary F, Flanagan AM (2019) An update of molecular pathology of bone tumors. Lessons learned from investigating samples by next generation sequencing. Genes Chromosom Cancer 58:88-99. https://doi.org/10.1002/gcc.22699

6. Fittall MW, Mifsud W, Pillay N, Ye H, Strobl AC, Verfaillie A, Demeulemeester J, Zhang L, Berisha F, Tarabichi M, Young MD, Miranda E, Tarpey PS, Tirabosco R, Amary F, Grigoriadis AE, Stratton MR, Van Loo P, Antonescu CR, Campbell PJ, Flanagan AM, Behjati S (2018) Recurrent rearrangements of FOS and FOSB define osteoblastoma. Nat Commun 9:2150. https://doi.org/10. 1038/s41467-018-04530-Z

7. Wagner EF, Eferl R (2005) Fos/AP-1 proteins in bone and the immune system. Immunol Rev 208:126-140. https://doi.org/10. 1111/j.0105-2896.2005.00332.x

8. van IJzendoorn DGP, de Jong D, Romagosa C, Picci P, Benassi MS, Gambarotti M, Daugaard S, van de Sande M, Szuhai K, Bovee JV (2015) Fusion events lead to truncation of FOS in epithelioid hemangioma of bone. Genes Chromosom Cancer 54:565-574. https:// doi.org/10.1002/gcc.22269

9. Huang SC, Zhang L, Sung YS, Chen CL, Krausz T, Dickson BC, Kao YC, Agaram NP, Fletcher CD, Antonescu CR (2015) Frequent FOS gene rearrangements in epithelioid hemangioma: a molecular study of 58 cases with morphologic reappraisal. Am J Surg Pathol 39:1313-1321. https://doi.org/10.1097/pas.0000000000000469

10. Walther C, Tayebwa J, Lilljebjorn H, Magnusson L, Nilsson J, von Steyern FV, Ora I, Domanski HA, Fioretos T, Nord KH, Fletcher CD, Mertens F (2014) A novel SERPINE1-FOSB fusion gene results in transcriptional up-regulation of FOSB in pseudomyogenic haemangioendothelioma. J Pathol 232:534-540. https://doi.org/10. 1002/path.4322

11. Antonescu CR, Chen HW, Zhang L, Sung YS, Panicek D, Agaram NP, Dickson BC, Krausz T, Fletcher CD (2014) ZFP36-FOSB fusion defines a subset of epithelioid hemangioma with atypical features. Genes Chromosom Cancer 53:951-959. https://doi.org/10. $1002 /$ gcc. 22206

12. Cleven AH, Hocker S, Briaire-de Bruijn I, Szuhai K, Cleton-Jansen AM, Bovee JVMG (2015) Mutation analysis of H3F3A and H3F3B as a diagnostic tool for giant cell tumor of bone and chondroblastoma. Am J Surg Pathol 39:1576-1583. https://doi. org/10.1097/pas.0000000000000512
13. Buddingh EP, Kuijjer ML, Duim RA, Burger H, Agelopoulos K, Myklebost O, Serra M, Mertens F, Hogendoorn PC, Lankester AC, Cleton-Jansen AM (2011) Tumor-infiltrating macrophages are associated with metastasis suppression in high-grade osteosarcoma: a rationale for treatment with macrophage activating agents. Clin Cancer Res 17:2110-2119. https://doi.org/10.1158/1078-0432.ccr10-2047

14. Meijer D, Gelderblom H, Karperien M, Cleton-Jansen AM, Hogendoorn PC, Bovee JV (2011) Expression of aromatase and estrogen receptor alpha in chondrosarcoma, but no beneficial effect of inhibiting estrogen signaling both in vitro and in vivo. Clin Sarcoma Res 1:5. https://doi.org/10.1186/2045-3329-1-5

15. Sundara YT, Kostine M, Cleven AH, Bovee JV, Schilham MW, Cleton-Jansen AM (2017) Increased PD-L1 and T-cell infiltration in the presence of HLA class I expression in metastatic high-grade osteosarcoma: a rationale for T-cell-based immunotherapy. Cancer Immunol Immunother 66:119-128. https://doi.org/10.1007/ s00262-016-1925-3

16. Hung YP, Fletcher CD, Hornick JL (2017) FOSB is a useful diagnostic marker for pseudomyogenic hemangioendothelioma. Am J Surg Pathol 41:596-606. https://doi.org/10.1097/pas. 0000000000000795

17. van IJzendoorn DGP, Sleijfer S, Gelderblom H, Eskens F, van Leenders G, Szuhai K, Bovee J (2018) Telatinib is an effective targeted therapy for pseudomyogenic hemangioendothelioma. Clin Cancer Res 24:2678-2687. https://doi.org/10.1158/10780432.ccr-17-3512

18. Hopman AHN, Ramaekers FCS (1998) Processing and staining of cell and tissue material for interphase cytogenetics. Curr Protocol Cytom 5:8.5.1-8.5.22

19. Franchi A, Calzolari A, Zampi G (1998) Immunohistochemical detection of c-fos and c-jun expression in osseous and cartilaginous tumours of the skeleton. Virchows Arch 432:515-519

20. Bussolati G, Leonardo E (2008) Technical pitfalls potentially affecting diagnoses in immunohistochemistry. J Clin Pathol 61:11841192. https://doi.org/10.1136/jcp.2007.047720

21. Bozec A, Bakiri L, Jimenez M, Schinke T, Amling M, Wagner EF (2010) Fra-2/AP-1 controls bone formation by regulating osteoblast differentiation and collagen production. J Cell Biol 190:1093-1106. https://doi.org/10.1083/jcb.201002111

22. Grigoriadis AE, Schellander K, Wang ZQ, Wagner EF (1993) Osteoblasts are target cells for transformation in c-fos transgenic mice. J Cell Biol 122:685-701

23. van IJzendoorn DGP, Forghany Z, Liebelt F, Vertegaal AC, Jochemsen AG, Bovee J, Szuhai K, Baker DA (2017) Functional analyses of a human vascular tumor FOS variant identify a novel degradation mechanism and a link to tumorigenesis. J Biol Chem 292:21282-21290. https://doi.org/10.1074/jbc.C117.815845

24. van IJzendoorn DGP, JVMG B (2017) Vascular tumors of bone: the evolvement of a classification based on molecular developments. Surg Pathol Clin 10:621-635. https://doi.org/10.1016/j.path.2017. 04.003

25. Harrington C, Accurso BT, Kalmar JR, Iwenofu OH, Agrawal A, Allen CM, Leon ME (2011) Aggressive osteoblastoma of the maxilla: a case report and review of the literature. Head Neck Pathol 5: 165-170. https://doi.org/10.1007/s12105-010-0234-y

26. Bertoni F, Unni KK, McLeod RA, Dahlin DC (1985) Osteosarcoma resembling osteoblastoma. Cancer. 55:416-426

Publisher's note Springer Nature remains neutral with regard to jurisdictional claims in published maps and institutional affiliations. 